\title{
Evaluación del acoplamiento ventrículo-arterial pulmonar mediante ecocardiografía Doppler
}

\section{Evaluation of the pulmonary ventricle-arterial coupling by Doppler echocardiography}

\author{
Tania Muñoz ${ }^{1 *}$, Elizabeth Hirschhaut ${ }^{1}$, Simón Tovar ${ }^{2}$, Rossana Quiñones ${ }^{1}$, Andrea Ovalles ${ }^{1}$ e \\ Yvette Betancourt $^{1}$ \\ ${ }^{1}$ Departamento de Cardiología, Unidad de Ecocardiografía; ${ }^{2}$ Departamento de Cardiología, Unidad de Hemodinamia. Hospital Militar Universitario \\ Dr. Carlos Arvelo, Caracas, Venezuela
}

\section{Resumen}

Antecedentes: El acoplamiento ventrículo-arterial (AVA) evalúa la función ventricular en respuesta al incremento de poscarga. En la hipertensión pulmonar (HP), el ventrículo derecho se adapta mejorando la contractilidad (acoplamiento) para mantener el flujo. El AVA sostiene el volumen latido preservando la eficiencia ventricular, luego se dilata manteniéndolo, pero con desacoplamiento y aumento de tensión de la pared. Para cuantificarlo se estima la relación elastancia ventricular, expresando la contractilidad miocárdica o presión isovolumétrica sistólica final, con la elastancia arterial, expresión de la carga independiente de la función ventricular, representada por la resistencia vascular pulmonar (RVP). Objetivo: Validar un nuevo índice ecocardiográfico, excursión sistólica del plano del anillo tricuspídeo (TAPSE)/RVP, como medida para evaluar la evolución del AVA en HP. Métodos: Estudio observacional y transversal dividido en dos etapas: a) en 10 pacientes validamos ecuaciones ecocardiográficas de presión media arterial pulmonar (PMAP) (Chemla), RVPa (resistencia vascular pulmonar según el algoritmo propuesto por Abbas) y RVPt (ecuación propuesta) comparando mediciones invasivas y no invasivas, empleando análisis de regresión lineal; b) se formaron dos grupos, $P M A P \leq 20 \mathrm{mmHg}(51)$ y $P M A P>20 \mathrm{mmHg}$ (51) con disfunción ventricular. Cuantificamos las variables ecocardiográficas: TAPSE, presión sistólica arterial pulmonar (PSAP), gradiente transtricuspídeo sistólico ( $\triangle R T)$, diámetro ventricular derecho (DVD) y RVP. Se estimaron los índices TAPSE/RVPa, TAPSE/RVPt y se compararon con TAPSE/PSAP e índice del producto de presión de contracción del ventrículo derecho (IPCVD), definido como TAPSE x $\triangle R T$. Empleamos análisis de regresión lineal y Bland-Altman. Resultados: Se reportó alta correlación entre los índices TAPSE/RVPa y TAPSE/RVPt, con TAPSE/PSAP (R²: 0.867 y 0.905, respectivamente). No aplicó el IPCVD en el grupo analizado. Conclusiones: Los índices ecocardiográficos TAPSE/RVP y TAPSE/PSAP SOn efectivos para la evaluar el AVA en HP.

Palabras claves: Resistencia vascular pulmonar. Hipertensión pulmonar. Presión media arterial pulmonar. Acoplamiento ventrículo-arterial. Elastancia arterial.

\section{Abstract}

Background: Ventricle-arterial coupling (VAC) evaluate the ventricular function in response to the increase post-load. In Pulmonary Hypertension (PH); the right ventricle (RV) adapts by improving contractility (coupling) to maintain flow. The VAC supports the stroke volume (SV) preserving ventricular efficiency, then dilates maintaining it but with decoupling and increased

Correspondencia:

*Tania Muñoz

E-mail: electroshock_2010@ hotmail.com
Disponible en internet: 22-06-2021 Rev Hosp Jua Mex. 2021;88(2):63-71 www.revistahospitaljuarez.com 1405-9622/@ 2021 Sociedad Médico-Quirúrgica del Hospital Juárez de México, A.C. Publicado por Permanyer. Este es un artículo open access bajo la licencia CC BY-NC-ND (http://creativecommons.org/licenses/by-nc-nd/4.0/). 
wall tension. To quantify it, the relationship between ventricular elastance is expressed by expressing myocardial contractility or final systolic isovolumic pressure (Ees/Pes-1) and arterial elastance (Ea) as an expression of the independent load of ventricular function, represented by pulmonary vascular resistance (PVR). Objective: To validate a new echocardiographic index: systolic excursion of the tricuspid ring plane (TAPSE)/RVP, as a measure to assess the evolution of VPA in HP. Methods: Observational, cross-sectional study divided into two phases; A)In 10 patients we validate echocardiographic equations of mean pulmonary arterial pressure (MPAP) (Chemla), PVRa (Abbas) and PVRt (proposed equation)comparing invasive and non-invasive measurements, using linear regression analysis. B)Two subgroups of patients were formed, MPAP $20 \mathrm{mmHg}(51)$ and $P M A P>20 \mathrm{mmHg}$ (51)and ventricular dysfunction (Vdf) We quantify the echocardiographic variables: TAPSE, SPAP, systolic transtricuspid gradient ( $\triangle T R$ ), right ventricle diameter (RVD) and PVR. TAPSE/PVRa, TAPSE/PVRt indexes were estimated and compared with TAPSE/SPAP and right ventricular contraction pressure product index (RVCPI), defined as TAPSEx: $\triangle T R$. We use linear regression analysis and Bland-Altman. Results: A high correlation was reported between the TAPSE/PVRa, TAPSE/ $P V R t$ indexes with TAPSE/SPAP $\left(R^{2}=0.867\right.$ and 0.905). Didn't apply RVCPI in the analyzed group. Conclusions: The echocardiographic indexes TAPSE/PVR and TAPS IPSAP are effective for the evaluation of VAC in PH.

Key words: Pulmonary vascular resistance. Pulmonary hypertension. Mean pulmonary arterial pressure. Ventricle-arterial coupling. Arterial elastance.

\section{Introducción}

El seguimiento del acoplamiento ventrículo-arterial (AVA) permite evaluar la adaptación del ventrículo derecho (VD) ante el aumento sostenido de la poscarga en la hipertensión pulmonar (HP). Su medición permite cuantificar la elastancia ventricular y arterial mediante el desarrollo de asas presión-volumen, posible con la técnica del latido único utilizando la resonancia magnética cardiaca (RMC) o colocando catéteres de conductancia en el VD con cateterismo cardiaco derecho $(C C D)^{1}$. La elastancia ventricular (Esf) es una medición de la contractilidad miocárdica o de la presión isovolumétrica al final de la sístole. La elastancia arterial (Ea), por su parte, cuantifica la carga independiente de la función ventricular representada por la resistencia vascular pulmonar (RVP), la presión sistólica arterial pulmonar (PSAP) y la presión media arterial pulmonar (PMAP) $)^{1}$. Guazzi, et al. ${ }^{2}$ propusieron el índice de excursión sistólica del anillo tricuspídeo (TAPSE)/PSAP para evaluar la función del VD, representando un indicador de la relación longitud/fuerza. Posteriormente Frea, et al. ${ }^{3}$ sugirieron que el índice del producto de contracción de ventrículo derecho (IPCVD) calculado mediante el gradiente de presión transvalvular tricuspídeo $(\Delta \mathrm{RT})$ x TAPSE representaba un modelo confiable para estimar el trabajo sistólico del VD, superando al índice TAPSE/PSAP. El TAPSE ha sido validado como uno de los métodos más útiles para evaluar la función sistólica del VD ${ }^{4-7}(4-7)$ aproximándonos por medio de la cuantificación de la longitud, al grado de respuesta de la fibra miocárdica sometida a la carga de presión durante toda la sístole.
El aumento de RVP expresa el cambio hemodinámico fundamental en la HP como consecuencia del remodelado vascular pulmonar. Su cálculo incluye el gradiente de presión en el sistema vascular pulmonar y volumen latido (VL) circulante por unidad de tiempo, permitiendo esta relación desarrollar asas presión-volumen vascular y cuantificar la Ea. Al incorporar el diámetro del ventrículo derecho (DVD) a la ecuación ecocardiográfica de RVP, obtendremos la representación de la piedra angular del acoplamiento, como lo es la dilatación del VD. En este trabajo proponemos: a) validar un nuevo índice ecocardiográfico TAPSE/RVP como método confiable y efectivo para la valoración del AVA en pacientes con HP, mediante la comparación con los índices TAPSE/PSAP e IPCVD previamente validados por sus autores; b) evaluar el rol y aporte del DVD en el AVA, comparando la ecuación RVPt con la $\mathrm{RVPa}$, que no lo incorpora.

\section{Métodos}

\section{Población y muestra}

Estudio observacional, transversal y prospectivo dividido en dos etapas. En la primera se incluyeron 10 pacientes referidos de los departamentos de cardiología y neumología para realizar CCD por probabilidad alta e intermedia de HP. Se cuantificaron PSAP, PMAP y RVP simultáneamente mediante ecocardiografía Doppler y CCD. Las ecuaciones ecocardiográficas empleadas fueron: Bernoulli, Chemla y Abbas, respectivamente. En la segunda etapa se formaron dos grupos de 51 pacientes cada uno a los que se les realizó ecocardiografía Doppler y cumplieron con los 
siguientes criterios de inclusión: a) pacientes con PMAP $\leq 20 \mathrm{mmHg}$, función diastólica normal o disfunción diastólica tipo 1 (relajación retardada) sin ninguna otra alteración; b) pacientes con mediciones de PMAP $>20 \mathrm{mmHg}$, disfunción ventricular sistólica y/o diastólica del ventrículo izquierdo (VI), ventrículo derecho (VD) o ambos. Se tomó el punto de corte de PMAP $>20 \mathrm{mmHg}$ para definir los dos grupos, por ser este el valor fijado como criterio diagnóstico de HP acordado por los expertos en el último Simposio Mundial de Hipertensión Pulmonar, celebrado en diciembre de 2018. Todos los pacientes fueron valorados en la sección de ecocardiografía del departamento de cardiología de nuestro centro, entre los meses de enero y marzo de 2019. Las características clínicas, demográficas y hemodinámicas de los pacientes con CCD de la primera parte del trabajo se muestran en la tabla 1. La categorización por patologías para el grupo de pacientes con PMAP $\geq 20 \mathrm{mmHg}$ (51), por cifras absolutas y porcentuales se reportan en la tabla 2. Las características demográficas y variables ecocardiográficas de ambos grupos (102) se registran en la tabla 3. Todos los pacientes firmaron consentimiento informado. El estudio fue aprobado por el Comité de Bioética y Ética Médica del hospital.

\section{Ecocardiografía}

Las mediciones Doppler y bidimensional se realizaron con los equipos Philips Sonos 7500 S3 y Philips iE33 SE1, de acuerdo a las guías de la Sociedad Americana de ecocardiografía y Asociación Europea de Imágenes Cardiovasculares ${ }^{4-7}$. La velocidad máxima de la regurgitación tricuspídea (VRT) se obtuvo por Doppler continuo colocado en el flujo regurgitante tricuspídeo a nivel valvular. Fue evaluado también en la vista cuatro cámaras. El $\triangle R T$ se obtuvo por la ecuación de Bernoulli $=4 \times V_{R T}^{2}$ y la PSAP $=\Delta R T+$ presión de la aurícula derecha $(P A D)^{8-10}$. La PAD se calculó según el diámetro y la variación respiratoria del diámetro la vena cava inferior $(\mathrm{VCl})$ : un diámetro de $\mathrm{VCl}<2.1 \mathrm{~cm}$ con colapso $>50 \%$ en inspiración indica PAD normal, de $3 \mathrm{mmHg}$ (intervalo: $0-5 \mathrm{mmHg}$ ), mientras que un diámetro de $\mathrm{VCl}>2.1 \mathrm{~cm}$ con colapso $<50 \%$ en inspiración profunda $0<20 \%$ con inspiración superficial representa una PAD de $15 \mathrm{mmHg}$ (rango: $10-20 \mathrm{mmHg}$ ). En los casos en los que el diámetro de la $\mathrm{VCl}$ o el colapso no encajó con estos parámetros se empleó un valor intermedio de $8 \mathrm{mmHg}$ (intervalo: $5-10 \mathrm{mmHg})^{4-6}$. Para la estimación de la función diastólica biventricular se utilizó Doppler pulsado (PW) en la vista apical de
Tabla 1. Características clínicas, hemodinámicas y demográficas de los pacientes con CCD $(n=10)$

\begin{tabular}{|c|c|}
\hline Sexo (M/F) & $5 / 5$ \\
\hline $\begin{array}{l}\text { Fracción de eyección } \\
\text { media (rango) \% }\end{array}$ & $55(30-75)$ \\
\hline $\begin{array}{l}\text { Presión sistólica arterial } \\
\text { pulmonar } \\
\text { Media (rango) mmHg }\end{array}$ & $42(27-101)$ \\
\hline $\begin{array}{l}\text { Presión capilar pulmonar mmHg } \\
\text { Media (rango) } \\
\leq 12 \mathrm{mmHg} \\
13-19 \mathrm{mmHg} \\
\geq 20 \mathrm{mmHg} \\
\text { RVPcat media (rango) (UW) } \\
\text { RVPeco media (rango) (UW) } \\
\text { GC (I/min) media (rango) } \\
\text { DVD media (rango) mm } \\
\text { ITVtsvd media (rango) cm }\end{array}$ & $\begin{array}{c}12(8-31) \\
8 \\
0 \\
2 \\
7.01(1.25-16.4) \\
5.29(1.64-13.33)^{*} \\
4(3.2-5.2) \\
34(22-55) \\
37.72(6-22)\end{array}$ \\
\hline $\begin{array}{l}\text { Diagnósticos } \\
\text { CIAOS HAP } \\
\text { Cardiopatía valvular tipo } \\
\text { insuficiencia mitral severa. HP } \\
\text { severa } \\
\text { EPOC. HP severa } \\
\text { Cardiopatía valvular aórtica y } \\
\text { mitral tipo doble lesión leve } \\
\text { CIV } \\
\text { Dilatación del tronco de la AP }\end{array}$ & $\begin{array}{l}2 \\
2 \\
3 \\
1 \\
1 \\
1\end{array}$ \\
\hline
\end{tabular}

CIAOS: comunicación interauricular tipo ostium secundum; CIV: comunicación interventricular; DVD: diámetro del ventrículo derecho; EPOC: enfermedad pulmonar obstructiva crónica; HAP: hipertensión arterial pulmonar; HP: hipertensión pulmonar; RVPcat: resistencia vascular pulmonar por cateterización; RVPeco: resistencia vascular pulmonar mediante ecocardiografía; GC: gasto cardíaco; ITVtsdv: integral tiempo-velocidad del tracto de salida del ventrículo derecho. *Cálculo obtenido por el algoritmo propuesto por Abbas: RVP eco $=$ RTV/ITVtsvd $x$ $10+0.16$, si RTV/ITVtsvd < 0.275; RTV²/ITVtsvd x 5, si RTV/ITVtsvd $\geq 0.275$.

cuatro cámaras. Se registró un volumen de muestra de 1 a $3 \mathrm{~mm}$ entre los extremos de las valvas de la válvula mitral y tricuspídea para medir el valor máximo de la velocidad del flujo (onda E) en la diástole temprana y la velocidad máxima de la onda $A$ en la diástole tardía, la relación $E / A$, tiempo de desaceleración de la onda E y tiempo de relajación isovolumétrica ${ }^{4-7}$. También se midió la relación $E / E^{\prime}$, la onda $E^{\prime}$ con el Doppler tisular colocado a nivel de la unión del anillo mitral (5-10 mm para cubrir el movimiento del anillo mitral en sístole y diástole) en los lugares de inserción septal y lateral de las valvas mitrales, obteniendo la velocidad E' septal y E' lateral ${ }^{4-6}$, promediando luego los valores obtenidos. La PMAP se estimó con la ecuación propuesta por Chemla $^{11}=0.61 \times$ PSAP +1.95 . El índice VRT/integral tiempo-velocidad del tracto de salida del ventrículo derecho (ITVtsvd) para calcular la RVP se determinó por ecocardiografía Doppler utilizando la vista del eje paraesternal corto a nivel de grandes vasos. La ITVtsvd 
Tabla 2. Categorización por patologías del grupo de pacientes con PMAP > $20 \mathrm{mmHg}(51)$, cifras absolutas y porcentajes

\begin{tabular}{|c|c|c|}
\hline Patologías & $\begin{array}{c}\text { N. }{ }^{\circ} \\
\text { pacientes }\end{array}$ & $\%$ \\
\hline $\begin{array}{l}\text { Cardiopatía mixta hipertensiva + } \\
\text { isquémica }\end{array}$ & 5 & 9.8 \\
\hline Cardiopatía valvular IMi severa & 4 & 7.84 \\
\hline Miocardiopatía dilatada & 1 & 1.96 \\
\hline $\mathrm{CIC}+\mathrm{MCP}$ & 2 & 3.92 \\
\hline CIC & 2 & 3.92 \\
\hline $\mathrm{CIC}$ en FD & 1 & 1.96 \\
\hline Cardiopatía hipertensiva & 10 & 19.6 \\
\hline Cardiopatía mixta ISQ + HAS + arritmia & 5 & 9.8 \\
\hline $\mathrm{EPOC}+\mathrm{HP}$ & 4 & 7.84 \\
\hline Cardiopatía arritmogénica & 2 & 3.92 \\
\hline Cardiopatía hipertensiva en FD & 8 & 15.68 \\
\hline Valvulopatía ruptura cuerda tricúspide & 1 & 1.96 \\
\hline Miocardiopatía restrictiva & 1 & 1.96 \\
\hline Cardiopatía mixta valvular+isquémica & 1 & 1.96 \\
\hline Estenosis mitral severa & 2 & 3.92 \\
\hline HAP idiopática & 1 & 1.96 \\
\hline CIV perimembranosa & 1 & 1.96 \\
\hline Total & 51 & 100 \\
\hline \multicolumn{3}{|c|}{$\begin{array}{l}\text { IMi: insuficiencia mitral; CIC: cardiopatía isquémica crónica; MCP: marcapaso } \\
\text { permanente; FD: fase dilatada; ISQ: isquémica; HAS: hipertensión arterial } \\
\text { sistémica; EPOC: enfermedad pulmonar obstructiva crónica; HP: hipertensión } \\
\text { pulmonar; HAP: hipertensión arterial pulmonar; CIV: comunicación interventricular; } \\
\text { PMAP: presión media arterial pulmonar. }\end{array}$} \\
\hline
\end{tabular}

(cm) se obtuvo con Doppler pulsado colocando el volumen de muestra a nivel del tracto de salida del ventrículo derecho (TSVD) de $5 \mathrm{~mm}$ proximal de la válvula pulmonar justo antes que comience el cierre ${ }^{4-7}$. La medición del diámetro del ventrículo derecho (DVD) en $\mathrm{mm}$ se hizo en el eje paraesternal largo evaluando el TSVD proximal o subvalvular medido desde el ángulo formado por la unión del anillo aórtico con el septum basal y la pared libre del VD al final de la diástole ${ }^{7}$. La RVP la calculamos con el algoritmo de Abbas, et al. ${ }^{12,13}$ : RVP = VRT / ITVtsvd $x 10+0.16$ si VRT / ITVtsvd $x 10$ $<0.275$ y VRT ${ }^{2}$ / ITVtsvd x 5 si VRT / ITVtsvd $\geq 0.275$; también con la ecuación propuesta en este trabajo: RVP = (VRT x DVD / ITVtsvd) $x$ fc (factor de corrección) ITVtsvd. El factor de corrección del ITVtsvd se estimó como sigue: si TVtsvd $\geq 10$ el fc ITVtsvd $=5$ y si
ITVtsvd $<10$ el fc ITVtsvd = ITVtsvd /2. La TAPSE se obtuvo midiendo en modo $\mathrm{M}$ la distancia en $\mathrm{mm}$ de la excursión del anillo, entre el final de la diástole y final de la sístole ${ }^{4-7}$. Se utilizó la herramienta del Doppler tisular para la estimación de velocidad sistólica del anillo tricuspídeo en la pared lateral del VD (VSt). La estimación de la fracción de eyección (FE) se determinó por el método de Simpson ${ }^{4-6}$. Las mediciones fueron realizadas tres veces y se promediaron.

\section{Cateterismo cardiaco derecho}

En la unidad de hemodinamia, a los pacientes del primer grupo (10) se les colocó un catéter de Swan Ganz arrow, 6 o $7 \mathrm{fr}$, para obtener las mediciones de presiones y flujos pulmonares. Se empleó la técnica de Seldinger en el abordaje de la vena yugular interna o subclavia ${ }^{14}$. El GC se determinó por la técnica de termodilución y la RVP con la ecuación: RVP = PMAP PCP/GC ${ }^{14-16}$. La presión capilar pulmonar (PCP) fue medida mediante la técnica de enclavamiento del balón por flotación y los valores de PMAP, PSAP, PDAP fueron registrados automáticamente por el equipo.

\section{Análisis estadístico}

Se utilizó MedCalc statistical software 2019 versión 18.11.3/14.0 de SPSS. Se realizó análisis estadístico descriptivo con estimación de promedio, mediana y desviación estándar (DS) de las diferentes variables ecocardiográficas cuantificadas en los pacientes de la segunda etapa representadas en la tabla 3. Se usó análisis de regresión lineal para comparar los 3 índices TASPE/RVP, TAPSE/PSAP y TASPEx $\triangle$ RT. Se modeló una ecuación y aplicó el análisis de Bland Altman para evaluar los límites de concordancia y media de las diferencias entre los métodos.

Las imágenes fueron reevaluadas para cuantificar la confiabilidad del intraobservador e interobservador.

\section{Resultados}

En el primer grupo analizado (tabla 1), 8 de ellos presentaron PCP igual o menor de $12 \mathrm{mmHg}$, la PSAP promedio fue $42 \mathrm{mmHg}$ y la RVPcat promedio fue de 7 UW. Según los subgrupos de la clasificación de HP aceptada por la Sociedad Española de Cardiología, las patologías estudiadas fueron:

- 1.4.4. Cardiopatía congénita: comunicación interauricular (CIA) (2), comunicación interventricular (CIV) (1). 
Tabla 3. Características demográficas y ecocardiográficas de los grupos de pacientes con PMAP $\leq 20 \mathrm{mmHg}$ y PMAP $>20 \mathrm{mmHg}$

\begin{tabular}{|c|c|c|c|c|c|c|}
\hline \multicolumn{4}{|c|}{ Pacientes con PMAP $\leq 20 \mathrm{mmHg}$} & \multicolumn{3}{|c|}{ Pacientes con PMAP > 20 mmHg } \\
\hline & Pmd (rango) & Mediana & DS & Pmd (rango) & Mediana & DS \\
\hline Edad & $38(18-57)$ & 36 & 7 & $65(25-83)$ & 66 & 12 \\
\hline $\operatorname{Sexo}(m / f)$ & $38 / 13$ & & & $31 / 20$ & & \\
\hline TAPSE (mm) & $20(24-18)$ & 20 & 1.7 & $15(8-25)$ & 15 & 4 \\
\hline PSAP $(\mathrm{mmHg})$ & $22(18-28)$ & 22 & 2.83 & $48(31-80)$ & 45 & 14 \\
\hline TAPSE/PSAP & $(0.71-1.22)$ & $0.92 / 0.93$ & 0.13 & $0.35(0.15-0.77)$ & 0.29 & 0.17 \\
\hline $\mathrm{E} / \mathrm{Ex}$ & $8(5-13)$ & 8 & 1.82 & $15(6-30)$ & 16 & 5 \\
\hline FE (\%) & $70(63-72)$ & 70 & 1.8 & $42(9-70)$ & 45 & 21 \\
\hline DVD (mm) & $27(22-32)$ & 27 & 1.7 & $36(22-55)$ & 33 & 7 \\
\hline VSt $(\mathrm{cm} / \mathrm{s})$ & $15(13-18)$ & 15 & 1 & $11(8-17)$ & 11 & 3 \\
\hline ITVtsvd (cm) & $17(16-19)$ & 17 & 0.7 & $12(6-19)$ & 12 & 4 \\
\hline RVPt (UW) & $2(1.5-2.4)$ & 1.61 & 0.23 & $(1.11-10.18)$ & $4.39 / 3.7$ & 2 \\
\hline TAPSE/RVPt & (8.8-14.86) & $12.54 / 12.25$ & 1.5 & $(1.2-13.41)$ & $5.0 / 4.0$ & 3 \\
\hline RVPa (UW) & $(1.21-1.66)$ & $1.41 / 1.39$ & 0.12 & $(1.12-11.42)$ & $4.41 / 2.66$ & 3 \\
\hline TAPSE/RVPa & $(11.76-18)$ & $15 / 15$ & 1.52 & $(1.25-18.55)$ & $5.85 / 4.91$ & 4 \\
\hline IPCvd (mmHg) & $(456-234)$ & $334 / 335$ & 70 & (216-1025) & $541 / 541$ & 186 \\
\hline VRT $(\mathrm{m} / \mathrm{s})$ & $(1.8-2.4)$ & 2 & 0.24 & $3(2.5-4)$ & 3 & 0.49 \\
\hline$\Delta \mathrm{RT}(\mathrm{mmHg})$ & $16(13-23)$ & 15 & 1.8 & $38(26-64)$ & 36 & 10 \\
\hline PAD (mmHg) & 5 & & & $10(5-20)$ & 10 & 5 \\
\hline
\end{tabular}

DS: desviación estándar; TAPSE: excursión sistólica del anillo tricuspídeo; PSAP: presión sistólica arterial pulmonar; PMAP: presión media arterial pulmonar; FE: fracción de eyección; DVD: diámetro del ventrículo derecho; VSt: velocidad sistólica del anillo tricuspídeo; ITVtsvd: integral tiempo velocidad del tracto de salida del ventrículo derecho; RVPt: resistencia vascular pulmonar según la ecuación propuesta en este trabajo; RVPa: resistencia vascular pulmonar según el algoritmo propuesto por Abbas; IPCvd: índice de presión de contracción del ventrículo derecho; VRT: velocidad máxima de la regurgitación tricuspídea; $\triangle R T$ : gradiente de regurgitación tricuspídea; PAD: presión de la aurícula derecha.

- 2.3. Valvulopatías: valvulopatía mitral tipo IM severa (2), valvulopatía aórtica tipo doble lesión aórtica y mitral (1).

- 3.1. Enfermedad pulmonar obstructiva crónica (EPOC) severa + HP severa (3).

- 3.5 Enfermedades del desarrollo pulmonar: dilatación del tronco de la arteria pulmonar (1).

Las patologías más representativas del grupo de pacientes con PMAP > $20 \mathrm{mmHg}$ y disfunción ventricu$\operatorname{lar}(\mathrm{dfV})$ fueron: cardiopatía hipertensiva no dilatada (10, $19.6 \%$ ), cardiopatía hipertensiva en fase dilatada ( 8 , $15.68 \%$ ) y cardiopatía mixta: isquémica e hipertensiva (5, 9.8\%) (Tabla 2). La edad promedio del grupo PMAP $\leq 20 \mathrm{mmHg}$ fue 38 (18-57) y del grupo PMAP > 20 $\mathrm{mmHg} 65$ (25-83); 38 pacientes fueron del sexo masculino (PMAP $\leq 20 \mathrm{mmHg}$ ) y 31 del grupo PMAP > $20 \mathrm{mmHg}$ (Tabla 3). La correlación obtenida cuando se compararon ambas ecuaciones de RVP (RVPa y RVPt) con los valores del CCD fueron altas $\left(R^{2}: 0.79\right.$ y $R^{2}$ : 0.84 , respectivamente) (Figs. 1 y 2 , parte superior). El análisis de Bland Altman mostró límites de concordancia satisfactorios entre los métodos: RVPa-CCD (media: 1.80; L: 6.83-3.22); RVPt-CCD (media: 2.02; L:6.982.73) (Figs. 1 y 2). Obtuvimos los valores de PMAP por ecocardiografía a partir de la fórmula propuesta por Chemla, et al.: PMAP $=0.61 \times$ PSAP + 1.95. Se encontró alta correlación con el análisis de regresión lineal entre el método invasivo y no invasivo $\left(R^{2}: 0.92\right.$, intervalo de confianza del $95 \%$ ), con límites de concordancia satisfactorios (media: 0.22; L: 1.52-1.09). Los métodos 


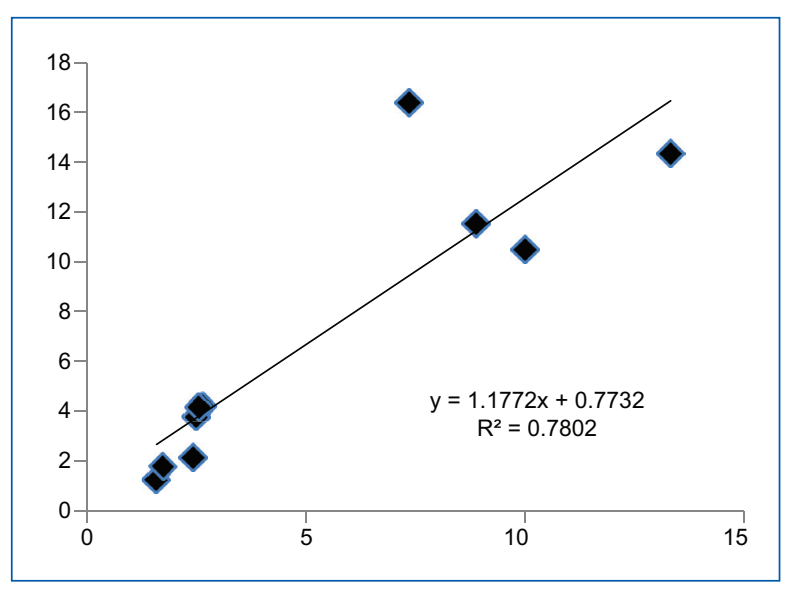

Figura 1. Análisis de regresión lineal entre la RVP obtenida por el algoritmo de Abbas y la estimada por CCD.

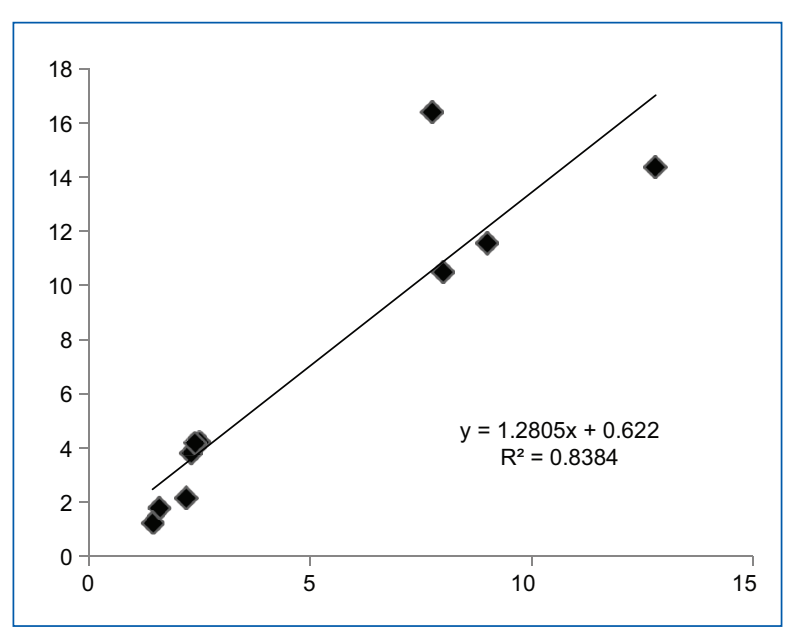

Figura 2. Análisis de regresión lineal entre la RVP obtenida por la ecuación de este estudio y la estimada por CCD.

ecocardiográficos TAPSE/PSAP y TAPSE/RVPt presentaron alta correlación en el grupo de 51 pacientes PMAP $\leq 20 \mathrm{mmHg}\left(\mathrm{R}^{2}:\right.$ 0.774) (Fig. 3), registrando una discreta menor correlación los métodos TAPSE/PSAP y TAPSE/RVPa (R²: 0.748) (Fig. 4). Al comparar los índices de TAPSE/PSAP y TAPSE/RVPt en el grupo de 51 pacientes con PMAP $>20 \mathrm{mmHg}$, se obtuvo muy alta correlación ( $R^{2}$ : 0.905) (Fig. 5), siendo menor la registrada entre los índices TAPSE/PSAP y TAPSE/ RVPa ( ${ }^{2}$ : 0.867) (Fig. 6). El análisis de Bland Altman en el subgrupo de 51 pacientes con PMAP $>20 \mathrm{mmHg}$ mostró una media de diferencia entre los métodos TAPSE/RVPa y TAPSE/RVPt de 2.156, con límites de concordancia satisfactorios, DS: 0.9 (4.07-0.241) (Fig. 7).

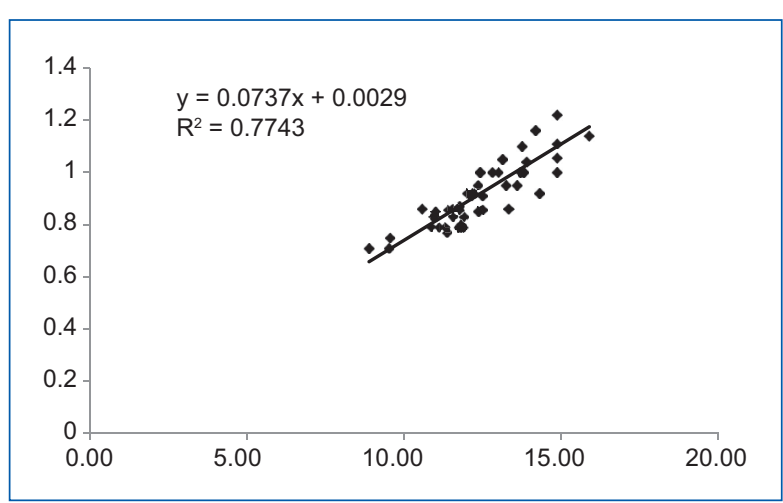

Figura 3. Análisis de regresión lineal entre la TAPSE/PSAP y la TAPSE/RVPt en el subgrupo de 51 pacientes sanos. TAPSE: excursión sistólica del anillo tricuspídeo; PSAP: presión sistólica arterial pulmonar; RVPt: resistencia vascular pulmonar según la ecuación propuesta en este trabajo.

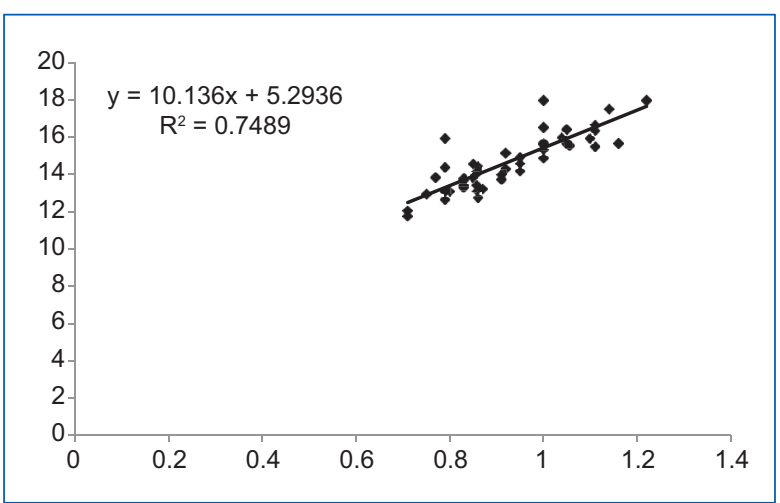

Figura 4. Análisis de regresión lineal entre la TAPSE/PSAP y la TAPSE/RVPa en el subgrupo de 51 pacientes sanos. TAPSE: excursión sistólica del anillo tricuspídeo; PSAP: presión sistólica arterial pulmonar; RVPa: resistencia vascular pulmonar según el algoritmo propuesto por Abbas.

\section{Discusión}

Las variables involucradas en el AVA: Esf, Ea y Esf/ $\mathrm{Ea}$, son estimadas con las asas presión-volumen mediante técnicas de RMC y catéter de conductancia en laboratorios de estudios especiales ${ }^{1,17}$. Poco se sabe de métodos ecocardiográficos para lograr ese propósito; Kuehne, et al..$^{17}$ realizaron un estudio en cerdos comparando métodos con cateterización derecha y RMC para validar las mediciones por este método. Al evaluar pacientes portadores de HP vs. grupo control, encontraron en el grupo enfermo una disminución de la función de bomba, mejoría de la contractilidad y AVA ineficaz. 


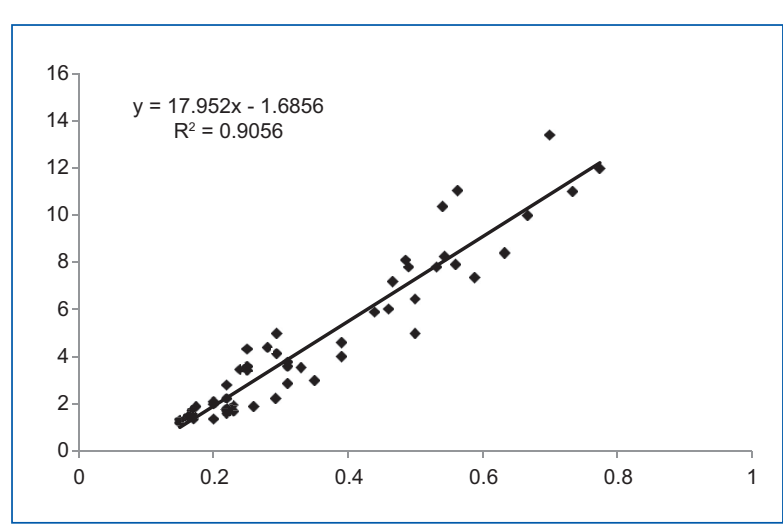

Figura 5. Análisis de regresión lineal entre la TAPSE/PSAP y la TAPSE/RVPt en el subgrupo de 51 pacientes con HP. TAPSE: excursión sistólica del anillo tricuspídeo; PSAP: presión sistólica arterial pulmonar; RVPt: resistencia vascular pulmonar según la ecuación propuesta en este trabajo; HP: hipertensión pulmonar.

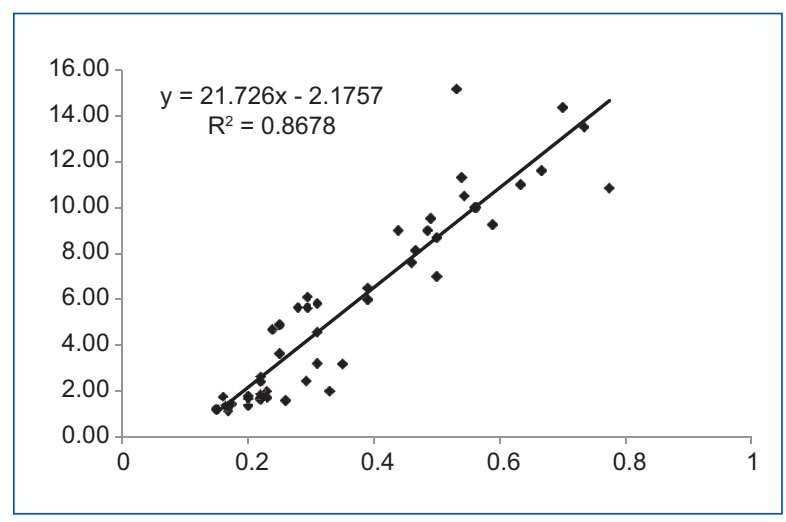

Figura 6. Análisis de regresión lineal entre la TAPSE/PSAP y la TAPSE/RVPa en el subgrupo de 51 pacientes con HP. TAPSE: excursión sistólica del anillo tricuspídeo; PSAP: presión sistólica arterial pulmonar; RVPa: resistencia vascular pulmonar según el algoritmo propuesto por Abbas; HP: hipertensión pulmonar.

Sanz, et al. ${ }^{18}$ realizaron CCD y RMC a un grupo de pacientes con HP, determinando la elastancia máxima (Emáx) y Ea como la relación PMAP/VSF y PMAP-PCP/ VL respectivamente, además de la relación Ea/Emáx. La Ea aumentó linealmente con el avance de la gravedad según lo definido por cuartiles de IRVP, mientras que la Emáx aumentó inicialmente y luego disminuyó; la relación Ea/Emáx se mantuvo temprana, pero aumentó notablemente con hipertensión grave, lo que indicó desacoplamiento Ea/Emáx. Vanderpool, et al. ${ }^{19}$ analizaron la relevancia clínica de las métricas de la adaptación funcional del VD al aumento de la poscarga.

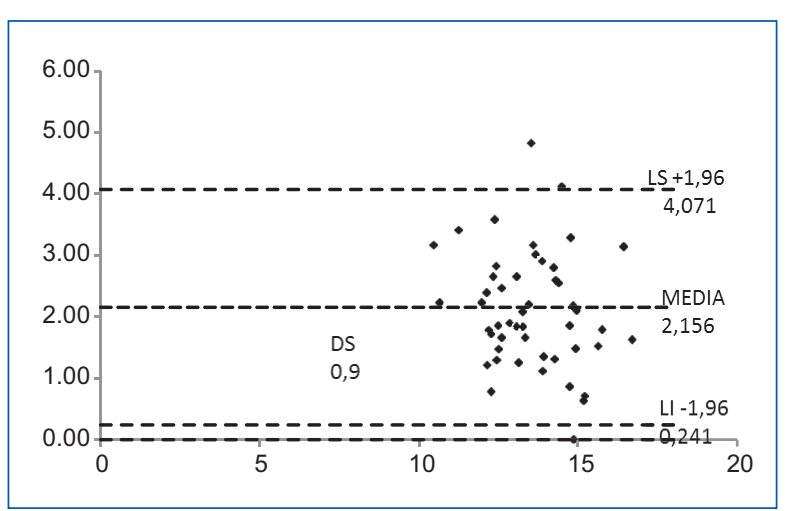

Figura 7. Análisis de Bland Altman entre la TAPSE/RVPa y la TAPSE/RVPt en el subgrupo de 51 pacientes con HP. TAPSE: excursión sistólica del anillo tricuspídeo; PSAP: presión sistólica arterial pulmonar; RVPt: resistencia vascular pulmonar según la ecuación propuesta en este trabajo; HP: hipertensión pulmonar; LS: límite superior; LI: límite inferior; DS: desviación estándar.

Lo midieron mediante la proporción VL/VSF, Pmáx/ PMAP o FE. La función diastólica del VD se estimó mediante el coeficiente de elastancia diastólica $(\beta)$. La supervivencia libre de trasplante se predijo por: PAD, PMAP, RVP, $\beta$, VL, VSF, VL/VSF y FEvd, pero después de controlar la presión auricular derecha, PMAP y VL; VL/VSF fue el único predictor independiente. Guazzi, et al. en su trabajo midieron con ecocardiograma la relación TAPSE/PSAP para determinar la función ventricular por medio del índice de la relación longitud/carga. Analizaron una población de pacientes con índice cardiaco (IC) y FE conservada y reducida, encontrando que un índice menor de 0.36 predice linealmente aumento de riesgo de eventos adversos (muerte/ complicaciones cardiovasculares) evaluando la función del VD más eficientemente sin verse afectada por la calidad de la disfunción del VI. En esta investigación encontramos la más alta correlación entre el índice TAPSE/PSAP con TASPE/RVPt y TAPSE/RVPa en la población enferma. Se registró un valor promedio/media para TAPSE/PSAP de 0.35/0.29 y TASPE/RVPt de 5/4. Frea, et al. realizaron un trabajo con 265 pacientes con clasificación NYHA (New York Heart Association) IV ingresados por descompensación aguda de insuficiencia cardiaca congestiva (ICC) avanzada (FE: $22 \pm 7 \%$; presión arterial sistólica: $107 \pm 20 \mathrm{mmHg}$ ); evaluaron predictores de puntos finales: muerte cardiaca, trasplante de corazón urgente e implantación de soporte circulatorio mecánico urgente a los 90 días. Concluyeron que el $\triangle \mathrm{RT}(<20 \mathrm{mmHg})$, el IPCVD $(<400 \mathrm{~mm} \times \mathrm{mmHg})$ y la $\mathrm{PAD}$ ( $\geq 20 \mathrm{mmHg}$ ) fueron los mejores predictores 
de resultados intrahospitalario y a corto plazo y que la HP no pudo predecir eventos. En esta investigación al comparar TAPSE/PSAP y TAPSE/RVP con IPCVD, este último método no aplicó al reportar baja correlación, posiblemente porque fueron incorporados pacientes con criterios de descompensación aguda en ICC avanzada, no representando el caso de la población analizada.

En la primera parte de este estudio, fueron validadas las ecuaciones ecocardiográficas de PSAP, PMAP, RVPa y RVPt, comparadas con las mediciones del CCD, reportando alta correlación en todos los casos. En la segunda parte, se evaluó el índice TAPSE/PSAP y comparamos con la propuesta de TAPSE/RVP. Se mantuvo la variable TAPSE en el índice de estimación de AVA, por ser un marcador indirecto ecocardiográfico del cambio de longitud desarrollada en la fibra miocárdica durante la sístole.

La RVP como componente del índice propuesto es la variable más fidedigna en la valoración de la Ea, expresión de la poscarga arterial producida por el remodelado vascular pulmonar en la $\mathrm{HP}^{1}$. Su estimación representa en el asa presión-volumen la relación existente entre las variaciones del gradiente de presión en el sistema vascular pulmonar y los cambios del volumen circulante por unidad de tiempo. En su medición se mantuvo el índice VRT/ITVtsvd (equivalente ecocardiográfico de $\Delta \mathrm{P} / \mathrm{Qp}$ ) e incorporó el DVD por ser el eje central en la valoración de la progresión del acoplamiento desde una etapa temprana (diámetro y contractilidad conservada), hasta la dilatación y falla ventricular al ser agotado el mecanismo compensador. Un trabajo publicado por Mohammed, et al. ${ }^{20}$, de la Clínica Mayo, evaluó el papel pronóstico de la disfunción del VD en un importante grupo de pacientes con insuficiencia cardiaca y fracción de eyección preservada (ICFEp). La función del VD fue evaluada mediante TAPSE en 500 de 562 pacientes y mediante evaluación semicuantitativa del tamaño del VD en toda la población. Se reportó que los pacientes con disfunción del VD leve o moderada a grave por evaluación semicuantitativa, en comparación con ninguna disfunción del VD, tuvieron más probabilidades de presentar fibrilación auricular, estimulación permanente y tratamiento diurético. Por el contrario, solo un tercil TAPSE más bajo reflejaba una condición clínica peor. En este estudio fue reportada mayor correlación entre RVPt y CCD que con RVPa y CCD del grupo de validación. También el grupo PMAP > 20mmHg, la correlación entre el índice TAPSE/PSAP y TAPSE/RVPt se reportó más alta que entre TAPSE/PSAP y TAPSE/RVPa. EI TAPSE se mantiene constante en todos los pacientes al comparar ambos métodos, demostrando que la incorporación el DVD es la responsable de la más alta correlación reportada en el grupo TAPSE/RVPt. El grupo PMAP $\leq 20 \mathrm{mmHg}$ también reportó mayor correlación entre el índice TAPSE/PSAP y TAPSE/RVPt que con TAPSE/PSAP y TAPSE/RVPa, sin embargo, los coeficientes de correlación registrados fueron más bajos que los reportados en el grupo de PMAP $>20 \mathrm{mmHg}$. Encontramos interesantes diferencias entre los métodos al analizar los valores de RVP cercanos al punto de corte de 3 UW (grupo de PMAP > $20 \mathrm{mmHg}$ ), como por ejemplo fue el caso de un subgrupo de 11 pacientes que reportaron RVPa $<3$ UW (2.3-2.8) vs. RVPt $\geq$ 3 UW (3-4.25), PMAP entre $26-38 \mathrm{mmHg}$, FE deprimida (14-45\%), con criterios disfunción del VD y relación $E / E^{\prime} \geq 15$ (15-30). Según los expertos, el patrón típico de HP-cardiopatía izquierda (grupo 2) por CCD, combina un PCP elevado (> $20 \mathrm{mmHg}$ ), PMAP levemente elevado (25-40 mmHg), y RVP que varía de $3 \mathrm{a}$ 4.9 UW, entre otras alteraciones hemodinámicas ${ }^{21}$. A pesar de que estas mediciones fueron obtenidas con CCD, no pasa desapercibida la semejanza encontrada con el grupo RVPt de este estudio.

La ecuación ecocardiográfica propuesta permite evaluar modificaciones graduales de DVD y ITVtsvd, y en consecuencia de la RVP; en cambio la RVPa entrega un amplio y abrupto margen de diferencias cuando Ilega al punto de corte definido por el algoritmo (VRT/ ITVtsvd $\geq 0.275$ ), pues la dispersión de valores deja de ser definida por una regresión lineal a tener un comportamiento de crecimiento exponencial.

\section{Recomendaciones}

Se recomienda el uso de estos sencillos y prácticos índices ecocardiográficos para la evaluar el AVA.

\section{Conclusiones}

Los índices TAPSE/RVP y TAPSE/PSAP son útiles y confiables en la evaluación del AVA para pacientes con HP.

La incorporación del DVD en la relación VRT/ITVtsvd optimiza la ecuación de RVP y el seguimiento de la evolución del AVA.

\section{Agradecimientos}

A la Dra. Rita Tamasaukas por su valioso aporte en la revisión metodológica de este manuscrito. Al equipo médico, técnicos en ecocardiografía y enfermería que 
laboran en los departamentos de cardiología del Hospital Militar Universitario Dr. Carlos Arvelo.

\section{Financiamiento}

Se utilizaron los recursos del hospital para realizar esta investigación.

\section{Conflicto de intereses}

Los autores informan que no hay ningún conflicto de intereses que declarar.

\section{Responsabilidades éticas}

Protección de personas y animales. Los autores declaran que los procedimientos seguidos se conformaron a las normas éticas del comité de experimentación humana responsable y de acuerdo con la Asociación Médica Mundial y la Declaración de Helsinki.

Confidencialidad de los datos. Los autores declaran que han seguido los protocolos de su centro de trabajo sobre la publicación de datos de pacientes.

Derecho a la privacidad y consentimiento informado. Los autores han obtenido el consentimiento informado de los pacientes y/o sujetos referidos en el artículo. Este documento obra en poder del autor de correspondencia.

\section{Bibliografía}

1. Vonk Noordegraaf $A$, Westerhof BE, Westerhof $N$. The relationship between the right ventricle and its load in pulmonary hypertension. J Am Coll Cardiol. 2017;69:236-43

2. Guazzi M, Bandera F, Pelissero G, Castelvecchio S, Menicanti L, Ghio S et al. Tricuspid annular plane systolic excursion and pulmonary arterial systolic pressure relationship in heart failure: an index of right ventricular contractile function and prognosis. Am J Physiol Heart Circ Physiol. 2013:305: H1373-H138.

3. Frea S, Pidello S, Bovolo V, lacovino C, Franco E, Pinneri F. Prognostic incremental role of right ventricular function in acute descompensation of advanced chronic heart failure. Eur J Heart Fail. 2016;18:564-72.
4. Lang RM, Badano LP, Mor-Avi V, Afilalo J, Armstrong A, Ernande L, et al. Recommendations for cardiac chamber quantification by echocardiography in adults: an update from the American Society of Echocardiography and the European Association of Cardiovascular Imaging. J Am Soc Echocardiogr. 2015;28:1-39.e14.

5. Lang RM, Badano LP, Mor-Avi V, Afilalo J, Armstrong A, Ernande L, et al. Recommendations for cardiac chamber quantification by echocardiography in adults: an update from the American Society of Echocardiography and the European Association of Cardiovascular Imaging. Eur Heart J Cardiovasc Imaging. 2015;16:233-70.

6. Mitchell C, Rahko P, Canaday B, Finstuen J, Foster M, Horton K, et al. Guidelines for performing a comprehensive transthoracic echocardiographic examination in adults: Recommendations from the American Society of Echocardiography. JASE. 2019;32(1):1-64.

7. Rudski L, Lai W, Afilalo J, Hua L, Handschumacher M, Chandrasekaran K, et al. Guildelines for the echocardiographic assessment of right heart in adults: A report from the American Society of Echocardiography, a registered branch of the European Society or Echocardiography. J Am Soc Echocardiogr. 2010;23(7):685-713.

8. Simonneau G, Montani G, Celermajer D, Denton C, Gatzoulis M, Krowka M, et al. Haemodynamic definitions and updated clinical classification of pulmonary hypertension. Eur Respir J. 2019;53(1):1801913.

9. Yock PG, Popp RL. Noninvasive estimation of right ventricular systolic pressure by Doppler ultrasound in patients with tricuspid regurgitation. Circulation. 1984;70:657-62.

10. Velazco L. Rol de la ecocardiografía en el estudio de la hipertensión arterial pulmonar. Avances Cardiol. 2009;29(2):154-64.

11. Chemla D, Castelain V, Humbert M, Hébert J, Simonneau G, Lecarpentier $Y$, et al. New formula for predicting mean pulmonary artery pressure using systolic pulmonary artery pressure. Chest. 2004;126:1313-8.

12. Abbas A, Fortuin D, Schiller N, Appleton C, Moreno C, Lester S. A simple method for noninvasive estimation of pulmonary vascular resistance. J. Am Coll Cardiol. 2003;41(6):1021-7.

13. Abbas AE, Franey L, Marwick T, Maeder M, Kaye D, Vlahos A, et al. Noninvasive assessment of pulmonary vascular resistance by Doppler echocardiography. J Am Soc Echocardiogr. 2014;27(1):108-9.

14. Reuter D, Huang C, Edrich T. Cardiac output monitoring using indicator-dilution techniques: basics, limits and perspectives. Anesth Analg. 2010;110:799-811.

15. David S. Celermajer echocardiographic and right heart catheterization techniques in patients with pulmonary arterial hypertension. Int J Cardiol. 2008:125(3):294-303.

16. Carrillo A, Fiol M, Rodríguez A. El papel del catéter de Swan-Ganz en la actualidad. Med Intens. 2010;34(3):203-14.

17. Kuehne $T$, Yilmaz S, Steendijk $P$, Moore $P$, Groenink $M$, Saaed $M$. Magnetic resonance imaging analysis of right ventricular pressure-volume loops: in vivo validation and clinical application in patients with pulmonary hypertension. Circulation. 2004;110:2010-6.

18. Sanz J, Garcia-Alvarez A, Fernandez-Friera L, Nair A, Mirelis J, Sawit S, et al. Right ventriculo-arterial coupling in pulmonary hypertension: a magnetic resonance study. Heart. 2012;98:238-43.

19. Vanderpool R, Pinsky M, Naeije R, Deible C, Kosaraju V, Bunner C, et al. RV-pulmonary arterial coupling predicts outcome in patients referred for pulmonary hypertension. Heart. 2015;101:37-43.

20. Mohammed S, Hussain I, Aboou O, Takahama H, Kwon S, Forfia P, at el. Right ventricular function in heart failure with preserved ejection fraction: A community based study. Circulation. 2014;130(25):2310-20.

21. Vachiéry J, Tedford J, Rosenkran S, Palazzin M, Lange I, Guazzi M, et al. Pulmonary hypertension due to left heart disease. Eur Respir J. 2019;53:1801897. 